\title{
Wiesse Rebagliati, Jorge Dante contempla la Trinità
}

Improvvisamente, mi resi conto che, con un'intuizione che andava in parallelo con quella dei grandi maestri italiani del XV e XVI secolo - tanto ammirati da Ricardo - ma con un linguaggio radicalmente contemporaneo, egli aveva creato un'opera che faceva diventare plastici ed evidenti gli arcani concetti filosofici e teologici che l'immaginazione esplosiva di Dante plasma nelle geometrie impossibili di quel portentoso canto, il XXXIII del Paradiso, l'ultimo della Commedia.

$\mathrm{I}^{1}$ testo di Jorge Wiesse Rebagliati, vincitore del $17^{\circ}$ Premio Internazionale Flaiano di Italianistica - La cultura italiana nel mondo, indaga un'inedita relazione tra Dante e l'arte peruviana contemporanea analizzando Dante contempla la Trinidad (2007) di Ricardo Wiesse,.

Nell'introduzione, intitolata Un Dante australe, l'autore racconta l'articolato iter che ha condotto all'elaborazione del testo. L'opera risale alla commemorazione dei 700 anni dal probabile inizio della stesura della Divina Commedia, avvenuta nell'aprile del 2007 su iniziativa del Dipartimento Accademico di Lettere e dell'Ufficio di Formazione Universitaria dell'Università del Pacífico di Lima, in Perú, del cui corpus docente è parte lo stesso Jorge Wiesse. Per il disegno del manifesto nel quale si annunciava la variegata gamma di attività culturali prevista per tale commemorazione gli organizzatori, ovvero l'autore stesso affiancato da Martina Vinatea Recoba e Carlos Gatti Murriel, si rivolsero al noto artista plastico Ricardo Wiesse. Come descritto nel testo, tale collaborazione fornì a Ricardo lo spunto per l'elaborazione di un'opera che si può considerare eccezionale all'interno della sua produzione. La singolarità dell'opera, data dalla sua complessità plastica e contenutistica, è tale da aver indotto Jorge Wiesse a tornare su di essa in diverse occasioni: prima, fra il 2012 e il 20I5, in una serie d'interventi che videro la sua partecipazione a simposi ed esposizioni dedicate all'opera dantesca dall'Università di Rochester, dalla medesima Università del Pacífico e a Biella, per l'inaugurazione della mostra L'America dantesca, curata da Luís Alfredo Agusti; e poi, nel 2017, dedicandole l'omonima pubblicazione Dante contempla la Trinità. 
Nel testo, l'autore descrive i tratti stilistici e tematici dell'opera, comparandola con gli aspetti caratteristici della produzione dell'artista. Da questa comparazione emerge tanto una continuità quanto una singolarità, che permette a Jorge Wiesse di individuare nell'opera in questione un importante traguardo: una sintesi emblematica della proposta di Ricardo che cristallizza il significato del canto dantesco. L'opera consiste in un oggetto tridimensionale che combina tecnica pittorica e scultorea sovrapponendo il profilo del poeta in rilievo, su modello del ritratto di Dante nella Disputa sul Santissimo Sacramento (I509) di Raffaello Sanzio, a un fondo oscuro e a un ovale che funge da cornice, entrambi intervenuti pittoricamente. Dal ritratto del poeta proposto dall'Urbinate nella Stanza della Segnatura Ricardo estrae una silhouette in filo d'argento, materiale tanto ricorrente nella sua produzione quanto lo stile gestuale e lineare che frequentemente l'artista utilizza per evocare il contesto al quale l'opera si riferisce. La linearità, prevalentemente curva, presente nello stile di Wiesse allude al territorio: da un lato, evoca la costa desertica peruviana, aspetto fortemente biografico e fondamentale per intendere la proposta artistica di Ricardo; dall'altro, riprende le espressioni culturali proprie del Perù precolombiano. Tali allusioni sono chiaramente illustrate nel testo, il quale è corredato da una selezione d'immagini che mostra il dialogo esistente tra l'opera di Ricardo e il contesto locale.

In quest'opera, territorio e cultura peruviani dialogano con il ritratto dantesco e con le immagini suggerite nel XXXIII canto del Paradiso. Lo sguardo contemplativo del poeta, sul quale la narrazione pone tanta enfasi, è il punto di partenza dell'analisi proposta da Wiesse. L'autore cita opportunamente la descrizione che Rocío Huatuco, dalla Pontificia Universidad Católica del Perú, fece dell'opera indicandola come un ritratto "attivo" per la direzione suggerita dall'occhio, che svia l'attenzione dal ritrattato per porre l'accento sull'atto contemplativo e sulla dimensione contemplata (Huatuco 20I7, pp. 2-3). Questo dettaglio permette all'artista di evidenziare il limite al quale giunge la descrizione verbale nel momento in cui il poeta contempla l'ineffabile. Terminata l'accurata descrizione dell'opera, Jorge Wiesse la compara con il canto della Commedia al fine di stabilire fino a che punto essa sviluppa il testo di riferimento, corredando tale comparazione di un vasto reticolato di rimandi letterari, filosofici e artistici.

Con quest'opera, Ricardo raccoglie la sfida insita nel voler descrivere un essere umano che contempla la divinità, interpretando brillantemente le metafore delle quali Dante si serve per descrivere i misteri dell'unità del molteplice, della Trinità e dell'Incarnazione alla fine del canto, le quali sono, rispettivamente: l'immagine di Dio come volume che racchiude ordinatamente i fogli squadernati che rappresentano l'infinita varietà dell'universo; i tre cerchi di diverso colore che ne formano uno solo, dove il secondo è riflesso del primo 
come l'arcobaleno mentre il terzo è descritto come una fiamma che spira dai primi due; e l'effige umana che a tratti collima con il secondo dei cerchi.

Va puntualizzato che il titolo completo del testo di Jorge Wiesse, che in copertina della presente edizione si propone in forma abbreviata, è in realtà il seguente: Dante contempla la Trinità (2007) di Ricardo Wiesse: un commento di Pd. XXXIII, IIS-I26? (Wiesse Rebagliati, 20I7, p. 23). Ragion per cui si potrebbe obiettare che i versi citati si riferiscono unicamente al mistero della Trinità, cadendo nell'equivoco di una forzatura interpretativa, se l'autore non si premurasse di spiegare l'intenzione contenuta nel punto interrogativo che chiude il titolo: mediante una domanda aperta, egli mette a disposizione del lettore l'ipotesi che l'opera, partendo dal mistero della Trinità descritto ai versi citati, colga nel contempo l'occasione fornita dalla relativa libertà del medium artistico per integrare nella sua peculiare interpretazione gli altri misteri trattati alla fine del canto in questione. Jorge Wiesse provvede inoltre a indicare la fonte di riferimento utilizzata dall'artista per quest'ultimo canto del Paradiso, ovvero l'edizione I Meridiani Mondadori curata da Anna Maria Chiavacci Leonardi (Chiavacci Leonardi, 1997, pp. 899-929), che nell'Introduzione al canto XXXIII e nelle note al testo illustra chiaramente il significato delle immagini proposte dal poeta per rappresentare i tre misteri contemplati. Tale fonte è stata tradotta per l'artista da Carlos Gatti, eminente dantista e collega dell'autore. I commenti di Gatti e Wiesse al canto, ai quali Ricardo si rivolse in fase d'elaborazione dell'opera, hanno altresì contribuito al risultato ottenuto.

L'occhio di Dante contempla, come si diceva, una dimensione superiore, che Ricardo evoca in uno sfondo oscuro attraversato da scie policrome simili a nebulose stellari che, nell'analisi dell'autore, rimandano al cosmo, ai cerchi della Trinità e all'unità del molteplice grazie alle suggestioni prodotte dal tratto e dalla varietà cromatica in esso presenti. L'autore fa inoltre notare come l'intersezione tra tale sfondo e il profilo del poeta sia particolarmente significativo in quanto plasma l'idea di una contemplazione interiore che si presta a molteplici interpretazioni che vanno dalla soggettivista, all'agostiniana, all'estatica. Quest'ultima interpretazione fornisce all'autore l'occasione per sviluppare ulteriormente la contestualizzazione storico-artistica dell'opera di Ricardo Wiesse, legandola alla tradizione artistica che va dal rinascimento e barocco italiano al Virreinato del Perú mediante una selezione di opere accomunate dal tema dell'estasi.

La densità di significato di Dante contempla la Trinità è tale da spingere l'autore a dedicare una riflessione ad altri dettagli salienti dell'artefatto, come l'ombra proiettata su sfondo e cornice dal filo d'argento che traccia il ritratto dantesco e la forma ovale dell'opera. Secondo Jorge Wiesse, la proiezione dell'ombra di Dante trasforma il soggetto in un ritratto universale, nella "nostra effige" ( $P d$. XXXIII, p. I3I) che riferisce l'umanità intera a Dante, come 
chiudendo il cerchio inaugurato dall'allusione alla "nostra vita" (If. I, pp. I-2) all'inizio del viaggio dantesco. In ultima istanza, e per mezzo del poeta, l'effige rimanda a Cristo, completando così il continuo rimando tra l'umanità, il poeta e la divinità che ne descrive il vincolo indissolubile e complesso. La proiezione della figura dantesca, che si riproduce circolarmente all'interno dell'opera, e la forma ovale della sua cornice permettono inoltre all'autore di stabilire un parallelismo con la circolarità immaginativa, linguistica e sonora del Paradiso.

Servendosi di un'analisi rigorosa e interdisciplinare, Jorge Wiesse ripercorre dunque gli aspetti salienti tanto dello stile e del contesto dell'artista quanto del canto dantesco di riferimento, individuando in Dante contempla la Trinità un trait d'union tra l'apoteosi del mondo dantesco contenuta nel XXXIII canto del Paradiso e l'arte contemporanea peruviana, emblematicamente rappresentata da Ricardo Wiesse e dall'opera in questione. L'autore dimostra con efficacia il grande valore di quest'ultima nel contesto della ricca tradizione iconografica di soggetto dantesco, data dalla capacità di evocare, in una sintesi resa felicemente possibile dal linguaggio delle arti figurative, il complesso insieme di significati e suggestioni descritto nell'ultimo canto della Commedia.

Giulia Degano

Universidad del Pacífico, Lima

Wiesse Rebagliati, Jorge

Dante contempla la Trinidad. Dante contempla la Trinità

Istituto Italiano di Cultura di Lima e Universidad Católica Sedes Sapientiae, Lima, 2017 